Article

\title{
A Rank-Ordered Marginal Filter for Deinterlacing
}

\section{Gwanggil Jeon ${ }^{1}$, Marco Anisetti ${ }^{2}$ and Seok Hoon Kang ${ }^{1, \star}$}

${ }^{1}$ Department of Embedded Systems Engineering, Incheon National University, 12-1 Songdo-dong, Yeonsu-gu, Incheon 406-772, Korea; E-Mail: gjeon@incheon.ac.kr

2 Dipartimento di Informatica, Università degli Studi di Milano, via Bramante 65 - 26013 Crema (CR), Italy; E-Mail: marco.anisetti@unimi.it

^ Author to whom correspondence should be addressed; E-Mail: hana@incheon.ac.kr; Tel.: +82-32-835-8422; Fax: +82-32-835-0782.

Received: 5 January 2013; in revised form: 13 February 2013 / Accepted: 16 February 2013 / Published: 4 March 2013

\begin{abstract}
This paper proposes a new interpolation filter for deinterlacing, which is achieved by enhancing the edge preserving ability of the conventional edge-based line average methods. This filter consists of three steps: pre-processing step, fuzzy metric-based weight assignation step, and rank-ordered marginal filter step. The proposed method is able to interpolate the missing lines without introducing annoying articles. Simulation results show that the images filtered with the proposed algorithm restrain less annoying pixels than the ones acquired by other methods.
\end{abstract}

Keywords: rank-ordered filter; adaptive marginal filter; deinterlacing; format conversion

\section{Introduction}

Interlaced scanning has been advanced from the early days of TV and still adopted for SDTV and 1080i HDTV broadcast standards [1]. However, nearly all late model flat panel displays (LCD, PDP, etc.) use progressive scanning formats. For these display devices, an entering interlaced video signal has to be transformed to a progressive one, and thus a scanning format conversion that gives compatibility between various video formats is required [2]. The super-resolution (SR) is a class of techniques that enhance the resolution of an imaging system [3-8]. The deinterlacing only considers vertical direction, while SR considers both of vertical and horizontal directions. Thus, the intra-field deinterlacing is a special case of SR. 
Many deinterlacing methods have been proposed, including spatial methods [9-13] and motion-based methods [14]. Although motion-based methods yield better subjective quality than spatial methods, they require reliable motion models and the estimated trajectories must be sufficiently proper, which generally causes excessive computational complexity. On the other hand, spatial methods have lower computational complexity since they only demand the current frame, making them more suitable for real-time applications. Therefore, in this paper, we focus on the spatial method.

Among spatial approaches, deinterlacing based on edge direction is the most outstanding and broadly adopted method. These methods calculate edge information first and then decide edge direction to utilize appropriate pixels for interpolation. Thus the edge information calculation and edge direction decision are the key steps. However, conventional methods have yielded poor performance when edge direction is not credible.

To shorten this issue, we propose a deinterlacing algorithm using rank-ordered fuzzy metric approach to reduce artifacts in deinterlaced images. In our approach, the missing lines are calculated by weight obtained using fuzzy metric $(F M)$ from the existing neighbor pixels. The local $F M$ infers the weight of the edge information. Thus, we deinterlace the interlaced signal without calculating edge directions as the traditional approaches do. After that, the rank-ordered differences statistic introduced in [15] is accommodated to the fuzzy context utilizing the introduced $F M$.

The paper is arranged as follows. Section 2 introduces $F M$ used in the weight assignation step. After that, the proposed filtering technique is described. Section 3 shows simulation results including performance comparison and computational complexity. Finally, conclusions are drawn in Section 4.

\section{Proposed Method}

\subsection{Fuzzy Metric for Weight Assignment}

A stationary $F M$, on a set $S$, is a fuzzy set of $S \times S$ satisfying the following conditions for all $p, q, r \in S[15]:$

$$
\begin{aligned}
& \text { Rule }_{1}: F M_{S}(p, q, t)>0 ; \\
& \text { Rule }_{2}: F M_{S}(p, q, t)=1 \text { if and only if } p=q ; \\
& \text { Rule }_{3}: F M_{S}(p, q, t)=F M_{S}(q, p, t) ; \\
& \text { Rule }_{4}: F M_{S}(p, q, t) \geq F M_{S}(q, r, u) * F M_{S}(p, r, t+u) ;
\end{aligned}
$$

where Rule $_{\sharp}$ is a $F M$ of rule number $\sharp$, * is a continuous $t$-norm, $F M_{S}(p, q, t)$ stands for the degree of nearness between $\mathrm{p}$ and $\mathrm{q}$ according to $\operatorname{Rule}_{2}, F M_{S}(p, q, t)$ is close to 0 when $p$ is far from $q$. Let $S$ be the set $\{0,1, \ldots, 255\}$, then, the function $F M: S \times S \rightarrow[0,1]$ given by

$$
F M_{S}(p, q)=\left(\frac{\min (p, q)+b}{\max (p, q)+b}\right)^{a}
$$

where $b$ is a small positive value for preventing $\max (p, q)=0$ singularity. As the difference between the components $p$ and $q$ become bigger, the value of $F M_{S}$ falls quickly. Thus, we assume $F M_{S}(p, q)$ is the fuzzy distance between the image components $p$ and $q$. Clearly, $F M$ is $F$-founded and it meets 


$$
0 \leq \frac{b}{I_{\max }+b} \leq F M_{S}(p, q) \leq 1
$$

for all $p, q \in S, I_{\max }$ is maximum pixel intensity, and $I_{\max }=255$ in this paper.

\subsection{Deinterlacing Implementation}

The proposed filter consists of three steps: (1) pre-processing step, (2) $F M$-based weight assignation step, and (3) rank-ordered marginal filter step. To begin with, we conduct interpolation with three missing pixels at location $(-1,0),(0,0)$, and $(1,0)$, with vertical six-tap filters. After that, we evaluate $F M$ degree using the introduced $F M$ equation. The obtained $F M$ degree is used for assigning weights. Finally, the missing pixel is calculated using the rank-ordered marginal filtering (ROMF) scheme.

Let us assume that $I$ is an image and $I_{(c, r)}$ is the pixel intensity at a position of $(c, r), c$ is column number and $r$ is raw number, and $I_{(0,0)}$ is the centered missing pixel to be processed. We denote $W$ as a filtering window centered on the pixel under processing of size $N \times N, N=3,5,7, \ldots$, which contains $n=N^{2}$ pixels. The pixels in $W$ are symbolized as $I_{(c, r)}$, and $c, r=-1,0,1$ for $N=3$ case.

The first step of the ROMF method is vertical six-tap filter (STF). This fixed coefficient six-tap Wiener filter is widely used to estimate the sub-pixels in video codec, such as MPEG-4, H.264/AVC, and some deinterlacing methods [16]. The coefficients of this filter can be different such as $h=[1,-5,20,20,-5,1] / 32$ or $h=[3,-17,78,78,-17,3] / 128$. In this paper, we chose the previous one for our system under the assumption that $h$ can calculate missing lines in the sub-pixel position properly. The missing pixels at $(c, 0)$ position, $c=-1,0,1$, are estimated using the adjacent pixels at $(c,-5),(c,-3),(c,-1),(c, 1),(c, 3)$, and $(c, 5)$, and we denote them as $I_{(c,-5)}, I_{(c,-3)}, I_{(c,-1)}$, $I_{(c, 1)}, I_{(c, 3)}$, and $I_{(c, 5)}$, respectively. To interpolate the pixel more precisely, we must adapt the filter to accommodate the new interpolation condition. Now, three pixels in the missing line $I_{(-1,0)}^{S T F}, I_{(0,0)}^{S T F}$, and $I_{(1,0)}^{S T F}$ are approximately deinterlaced applying Equation (3); however, they are not the same with the original missing pixel. Figure 1 shows the pixel positions with filter coefficients.

$$
I_{(c, 0)}^{S T F}=h(1) I_{(c,-5)}+h(2) I_{(c,-3)}+h(3) I_{(c,-1)}+h(4) I_{(c, 1)}+h(5) I_{(c, 3)}+h(6) I_{(c, 5)}
$$

Figure 1. The pixel positions with filter coefficients.

$$
\left.\begin{array}{lllllll}
a & b & c & d & e & f
\end{array}\right]
$$


For the ROMF, eight neighboring pixels, $I_{(-1,-1)}, I_{(0,-1)}, I_{(1,-1)}, I_{(-1,0)}^{S T F}, I_{(1,0)}^{S T F}, I_{(-1,1)}, I_{(0,1)}$ and $I_{(1,1)}$ are employed to deinterlace the missing center pixel at $(0,0)$. In this paper, we take $F M_{S}$ as the distance function (note, however, that any other function such as Euclidean distance could be used). Therefore, the distance between two pixels $I_{(0,0)}^{S T F}$ and $I_{(c, r)}$ is symbolized as $F M_{S}\left(I_{(0,0)}^{S T F}, I_{(c, r)}\right)$. We denote $\bar{W}$ the set of neighbors of $I_{(0,0)}^{S T F}$, that is, $\bar{W}=W-I_{(0,0)}^{S T F}$.

The second step is to calculate eight $F M$ using $F M_{S}\left(I_{(0,0)}^{S T F}, I_{(c, r)}\right)$ where $I_{(c, r)} \in \bar{W}$. The proposed deinterlacing solves the problem by looking for the most robust $I_{(c, r)}$ pixel. To compute ROMF, the distance $F M_{S}\left(I_{(0,0)}^{S T F}, I_{(c, r)}\right)$ are rearranged in an ascending order so that a group of non-negative real values $\chi_{m}$, where fixed a positive integer $m \leq n-1$, are obtained. Note that $\chi_{m}$ is not always different: $\chi_{1} \leq \chi_{2} \leq \ldots \leq \chi_{m} \leq \ldots \chi_{n-1}$. Generally speaking, $\chi_{j}$ is the $j^{\text {th }}$ smallest $F M_{S}\left(I_{(0,0)}^{S T F}, I_{(c, r)}\right)$ value, and its associated $I_{(c, r)}$ is denoted as $I_{\chi_{j}}$. Finally, the proposed ROMF calculates the missing pixel $I_{(0,0)}^{R O M F}$ :

$$
I_{(0,0)}^{R O M F}=\frac{1}{2} I_{(0,0)}^{S T F}+\frac{1}{2} \frac{\sum_{j=-1}^{m} \chi_{j} \cdot I_{\chi_{j}}}{\sum_{j=-1}^{m} \chi_{j}}
$$

where $\chi_{j}$ is assumed to be a weight factor. It can be observed from Equations (1) and (4) that, when $I_{\chi_{j}}$ and $I_{(0,0)}^{S T F}$ have similar values, the weight factor $\chi_{j}$ becomes large. On the other hand, when the difference between $I_{\chi_{j}}$ and $I_{(0,0)}^{S T F}$ are large, $\chi_{j}$ becomes smaller. Thus, the missing line is deinterlaced based on the similarity among their neighbor pixels, $I_{\chi_{j}}$, with allocated weights based on the $F M$.

\section{Simulation Results}

To evaluate the performance of the proposed algorithm, we present the simulation results in this section. We considered twenty images and video sequences as the dataset, which are shown in Table 1. The ten images starting with "A" to "G" are the test images, and the others (images starting with "K" to "Z") are the training images.

Table 1. Test and training sets classified by alphabetical order.

\begin{tabular}{|}
\hline Test images (I) and video (V) sequences (images starting with "A" to "G”): \\
\hline Airplane (I), Akiyo (V), Barbara (I), Bluesky (V), Boat (I), \\
Bus (V), City (V), Finger (I), Football (V), Girl (I) \\
\hline Training images (I) and video (V) sequences (images start with "K" to “Z”): \\
\hline Kimono (V), Lena (I), Man (I), Milkdrop (I), Mobile (V), \\
News (V), Peppers (I), Raven (V), Toys (V), Zelda (I) \\
\hline
\end{tabular}

We conducted simulation using MATLAB with an Intel(R) Core(TM) i5 CPU M460 @ $2.53 \mathrm{GHz}$ processor. We compared the proposed method with MELA [9], LABI [10], FEPD [11], MCAD [12] and LSMD [13] methods. Note that the designed filter parameters $a$ and $b$ and the number of considered neighbor pixels $m$ play crucial roles, making it important to set them appropriately. One assumption is that, as we mentioned in Section 2, parameter $b$ is a small positive value for avoiding $\max (p, q)=0$ singularity. Thus we gave $b=1$, which is the smallest intensity step. Figure 2 shows the average MSE 
performance of the proposed method according to various $m$ values under the condition of $b=1$ and $1 \leq a \leq 15$. From Figure 2, $m=3$ is determined to give the least MSE. Another parameter $a=10$ is determined under the condition of $b=1$ and $m=3$, as shown in Figure 3.

Figure 2. Performance of the proposed method in terms of MSE as a value of $m$ under the condition of $b=1$, for $1 \leq a \leq 15$.

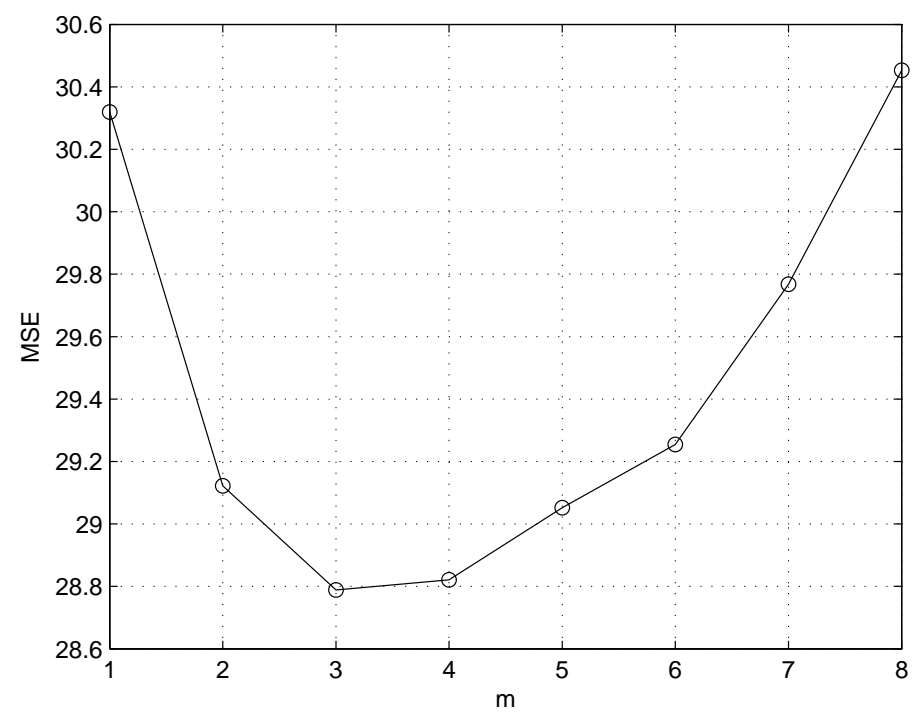

Figure 3. Performance of the proposed method in terms of MSE as a value of $a$ under the condition of $b=1$ and $m=3$.

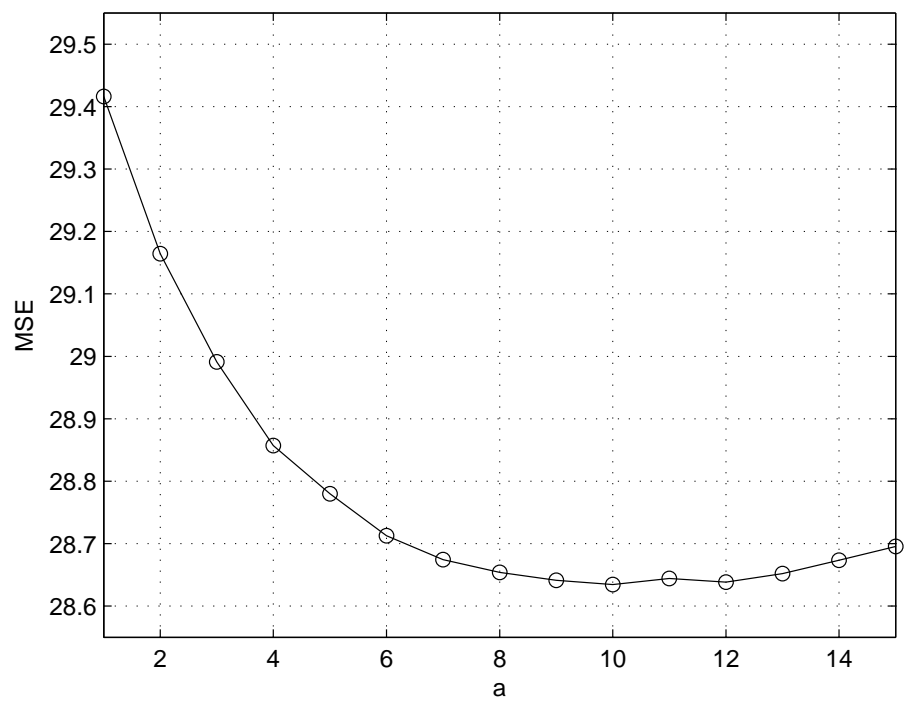

The PSNR metric in decibels $(\mathrm{dB})$ was selected to evaluate the performance. Table 2 shows the comparison results of the PSNR performance of the proposed method to the benchmarks. After the experiments, it is obvious that the proposed method outperforms other methods by 0.959 (MELA), 1.199 (LABI), 2.414 (FEPD), 1.541 (MCAD), and 1.377 (LSMD) dB in terms of average PSNR. For Akiyo and Bus image, MELA showed a better PSNR performance of $0.107 \mathrm{~dB}$ and $0.035 \mathrm{~dB}$. However, the proposed method showed the best PSNR performance for the other images.

Table 3 shows the CPU time per image. As we can see, the proposed method has more complexity than MELA. However, the proposed technique reduces the average CPU time up to $93.74 \%, 96.89 \%$, 95.57\%, and 79.52\% when compared with LABI, FEPD, MCAD, and LSMD, respectively. 
Table 2. Comparison of the average PSNR for 10 test images and video sequences among different deinterlacing methods.

\begin{tabular}{|c|c|c|c|c|c|c|c|}
\hline & MELA & LABI & FEPD & MCAD & LSMD & ROMF & Ranking \\
\hline airplane & 35.088 & 35.345 & 34.385 & 35.085 & 35.660 & 36.084 & 1 \\
\hline akiyo & 40.205 & 38.841 & 37.255 & 39.726 & 38.149 & 40.098 & 2 \\
\hline barbara & 32.018 & 31.930 & 28.879 & 25.929 & 29.414 & 33.562 & 1 \\
\hline bluesky & 37.900 & 37.798 & 37.510 & 38.107 & 39.373 & 39.547 & 1 \\
\hline boat & 35.186 & 35.277 & 33.074 & 35.342 & 33.762 & 36.034 & 1 \\
\hline bus & 28.654 & 28.217 & 28.104 & 28.262 & 28.095 & 28.619 & 2 \\
\hline city & 31.460 & 31.497 & 31.258 & 31.527 & 31.656 & 31.726 & 1 \\
\hline finger & 31.323 & 31.362 & 30.679 & 31.810 & 32.085 & 32.946 & 1 \\
\hline football & 35.057 & 34.475 & 33.308 & 35.034 & 34.763 & 35.791 & 1 \\
\hline girl & 41.793 & 41.535 & 39.676 & 42.038 & 41.545 & 43.861 & 1 \\
\hline avg. & 34.868 & 34.628 & 33.413 & 34.286 & 34.450 & 35.827 & 1 \\
\hline
\end{tabular}

Table 3. Comparison of the average CPU time for 10 test images and video sequences among different deinterlacing methods.

\begin{tabular}{|c|c|c|c|c|c|c|c|}
\hline & MELA & LABI & FEPD & MCAD & LSMD & ROMF & Ranking \\
\hline airplane & 0.547 & 14.698 & 28.491 & 22.547 & 4.286 & 1.231 & 4 \\
\hline akiyo & 0.207 & 5.694 & 10.947 & 7.861 & 1.838 & 0.596 & 4 \\
\hline barbara & 0.490 & 14.154 & 29.527 & 20.609 & 4.409 & 0.787 & 4 \\
\hline bluesky & 3.076 & 123.768 & 228.331 & 159.369 & 34.895 & 6.067 & 4 \\
\hline boat & 0.470 & 13.490 & 28.727 & 20.191 & 4.076 & 1.107 & 4 \\
\hline bus & 0.164 & 5.406 & 10.919 & 8.846 & 2.304 & 0.703 & 4 \\
\hline city & 1.358 & 48.484 & 105.553 & 73.752 & 16.502 & 2.590 & 4 \\
\hline finger & 0.397 & 12.349 & 29.005 & 20.315 & 4.036 & 1.057 & 4 \\
\hline football & 0.203 & 5.057 & 12.676 & 7.755 & 1.668 & 0.794 & 4 \\
\hline girl & 0.433 & 12.305 & 30.801 & 19.886 & 4.070 & 1.065 & 4 \\
\hline avg. & 0.734 & 25.54 & 51.498 & 36.113 & 7.809 & 1.599 & 4 \\
\hline
\end{tabular}

The Barbara image in Figure 4 has many low and high-angle directions that the previous methods may miss. Figure $4(\mathrm{a}-\mathrm{g})$ shows poor performance because only a limited number of edge directions 
were utilized, which does not compensate for inaccurate edge information. Figure 4(h,i) shows better results than the other conventional methods. However, the diagonal edge reconstruction is not sufficient. The proposed method, however, performs well for this case as shown in Figure 4(j). Figure 5 shows the results for the Boat image. The result for this image also shows that the proposed method is superior to other methods.

Figure 4. Comparison of subjective qualities in Barbara image: (a) original Barbara; (b) MELA; (c) LABI; (d) FEPD; (e) MCAD; (f) LSMD; and (g) ROMF.

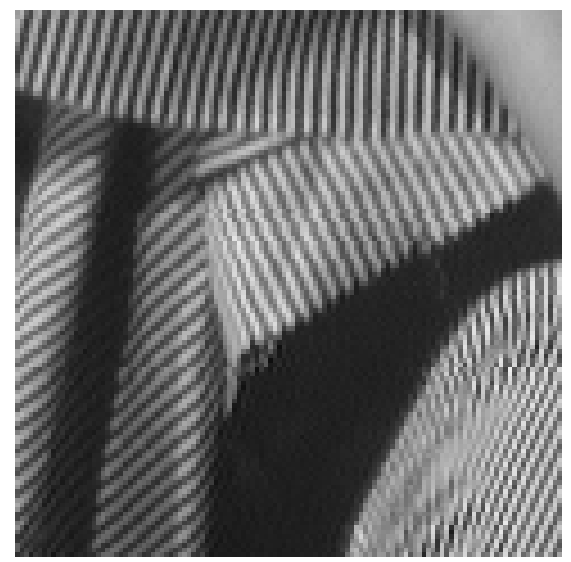

(a)

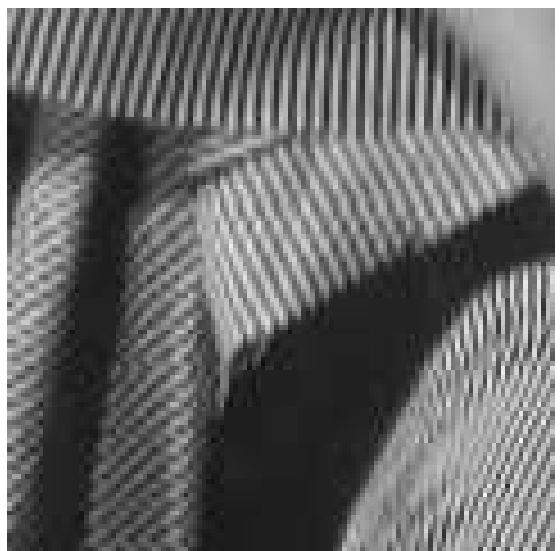

(b)

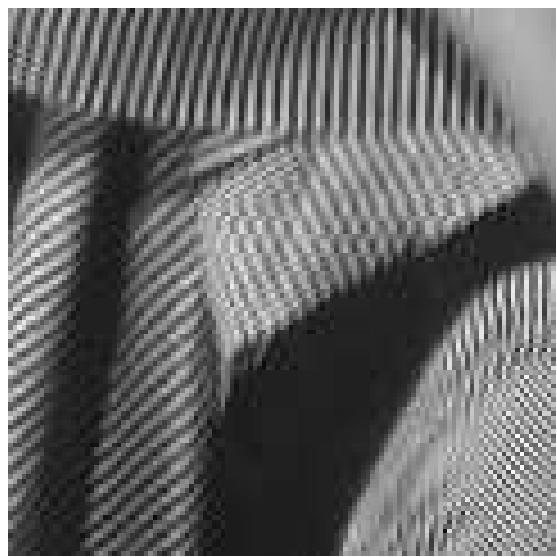

(e)

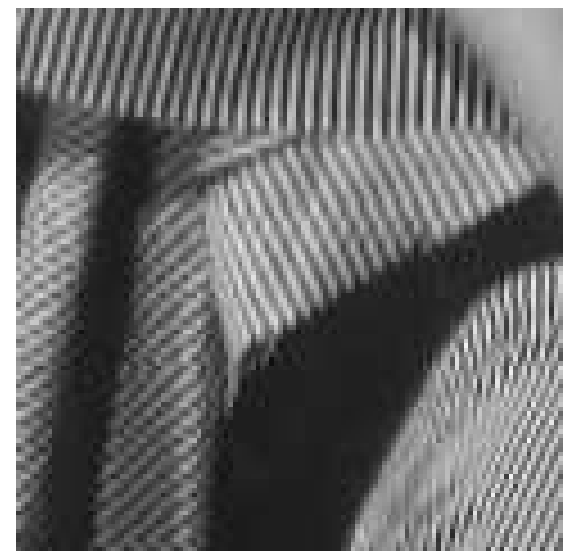

(c)

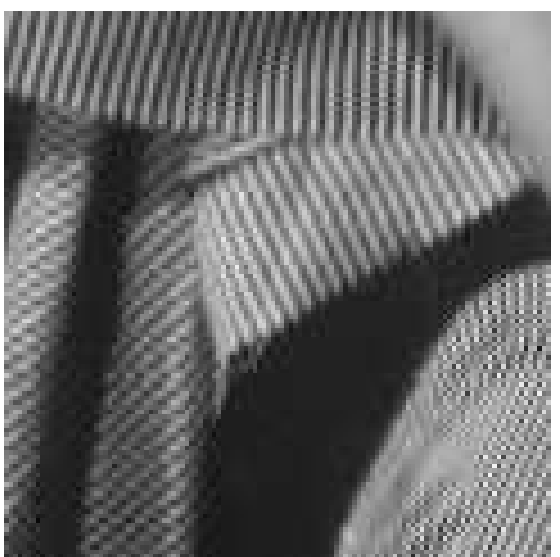

(f)

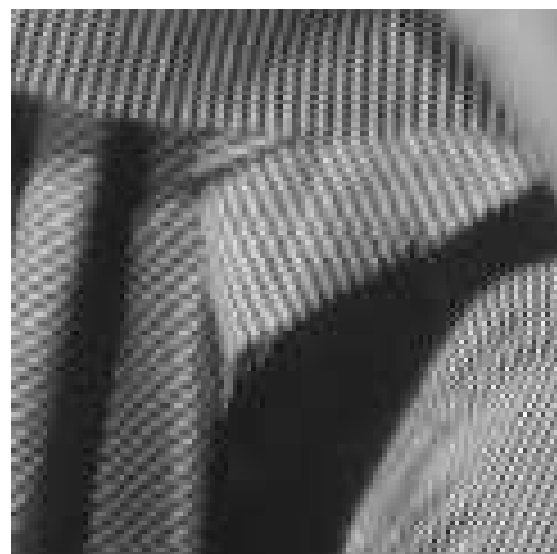

(d)

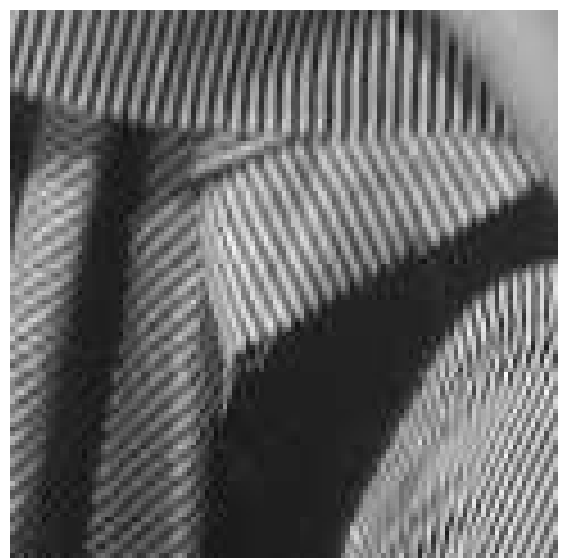

(g) 
Figure 5. Comparison of subjective qualities in Boat image: (a) original Boat; (b) MELA; (c) LABI; (d) FEPD; (e) MCAD; (f) LSMD; and (g) ROMF.

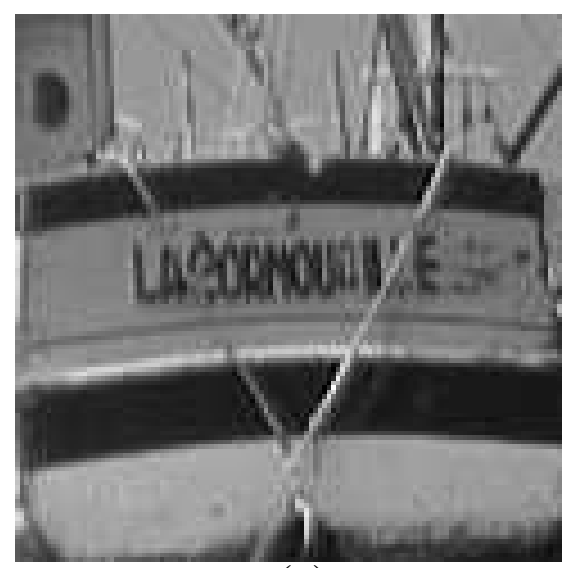

(a)

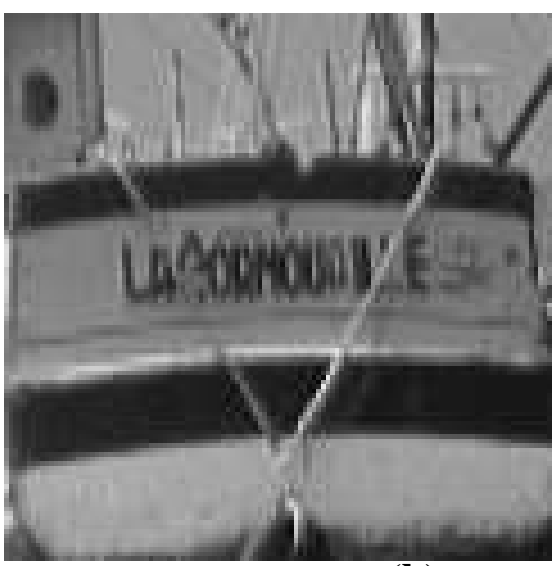

(b)

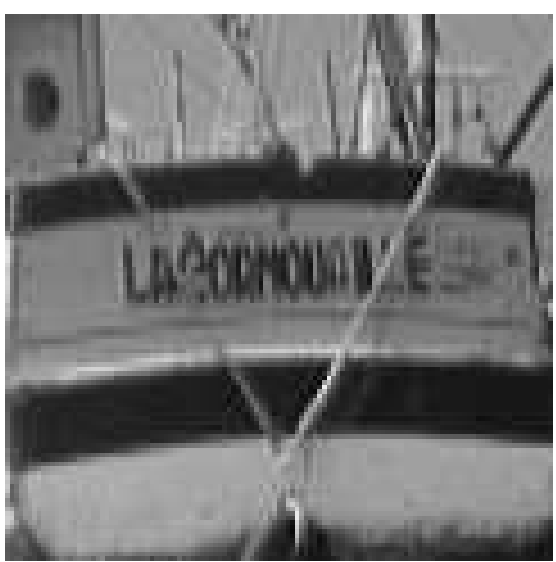

(e)

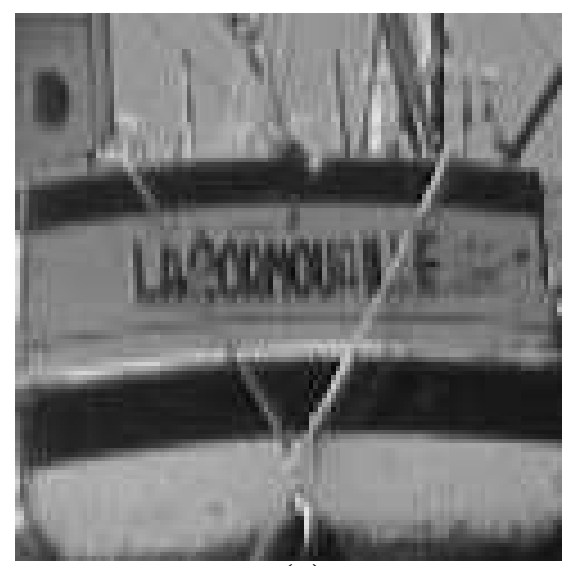

(c)

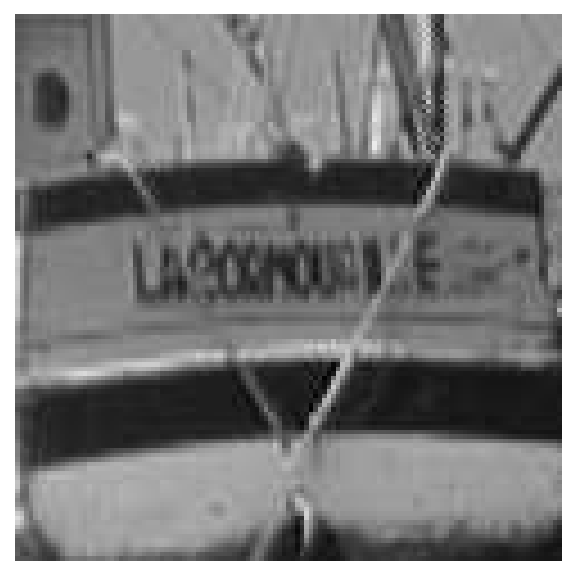

(f)

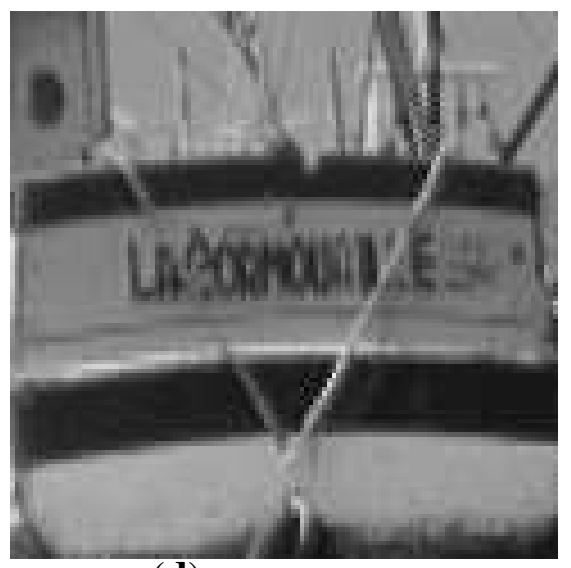

(d)

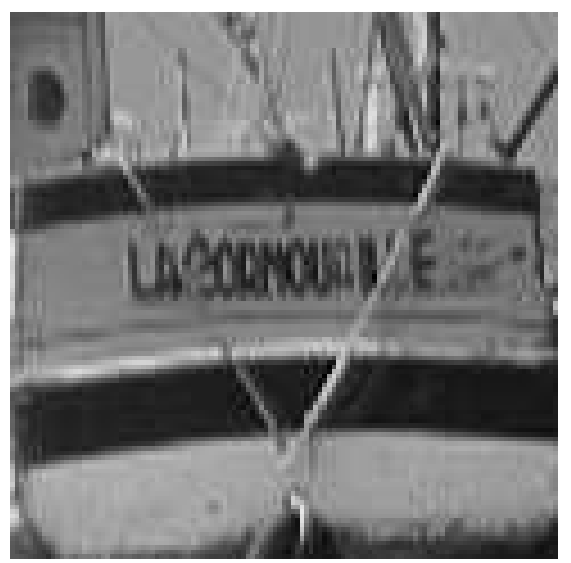

(g)

\section{Conclusions}

This paper presented an effective spatial deinterlacing method, which is achieved by improving the edge preserving ability of the conventional edge-based line average method. This filter consists of three steps: pre-determined six-tap filter based pre-processing step, $F M$-based weight assignation step, and rank-ordered marginal filter step. The experimental results indicated that ROMF has achieved these two 
goals and has promising performance subjectively and objectively. Meanwhile, ROMF has merits of low complexity for real-time application.

\section{Acknowledgments}

This work was supported by the Incheon National University Research Grant in 2012.

\section{References}

1. Jack, K. Video Demystified-A Handbook for the Digital Engineer, 4th ed.; Elsevier: Oxford, UK, 2005.

2. Bellars, E.B.; Haan, G.D. Deinterlacing: A Key Technology for Scan Rate Conversion; Elsevier: Oxford, UK, 2000.

3. Wu, J.; Paul, A.; Xing, Y.; Fang, Y.; Jeong, J.; Jiao, L.; Shi, G. Morphological dilation image coding with context weights prediction. Signal Process. Image Commun. 2010, 25, 717-728.

4. Wu, W.; Liu, Z. Learning-based super resolution using kernel partial least squares. Image Vis. Comput. 2011, 29, 394-406.

5. Wu, J.; Huang, J.; Jeon, G.; Jeong, J.; Jiao, L.C. An adaptive autoregressive de-interlacing method. Opt. Eng. 2011, 50, doi:10.1117/1.3572125.

6. Wu, J.; Liu, Z.; Gueaieb,W.; He, X. Single-image super-resolution based on Markov random field and contourlet transform. J. Electron. Image 2011, 20, doi:10.1117/1.3580750.

7. Wu, J.; Li, T.; Hsieh, T.-J.; Chang, Y.-L.; Huang, B. Digital signal processor-based 3D wavelet error-resilient lossless compression of high-resolution spectrometer data. J. Appl. Remote Sens. 2011, 5, doi:10.1117/1.3663955.

8. Wu, J.; Liu, Z.; Krys, D. Improving laser image resolution for pitting corrosion measurement using markov random field method. Autom. Constr. 2012, 21, 172-183.

9. Kim, W.; Jin, S.; Jeong, J. Novel intra deinterlacing algorithm using content adaptive interpolation. IEEE Trans. Cons. Electron. 2007, 53, 1036-1043.

10. Lee, D.-H. A new edge-based intra-field interpolation method for deinterlacing using locally adaptive-thresholded binary image. IEEE Trans. Cons. Electton. 2008, 54, 110-115.

11. Yang, S.; Kim, D.; Jeong, J. Fine edge-preserving deinterlacing algorithm for progressive display. IEEE Trans. Cons. Elect. 2009, 55, 1654-1662.

12. Park, S.J.; Jeon, G.; Jeong, J. Covariance-Based Adaptive Deinterlacing Method Using Edge Map. In Proceedings of the IEEE IPTA2010, Paris, France, July 2010; pp. 166-171.

13. Park, S.J.; Jeong, J. Local surface model-based deinterlacing algorithm. Opt. Eng. 2011, 50, doi: 10.1117/1.3533027.

14. Jeon, G.; Anisetti, M.; Kim, D.; Bellandi, V.; Damiani, E.; Jeong, J. Fuzzy rough sets hybrid scheme for motion and scene complexity adaptive deinterlacing. Image Vis. Comput. 2009, 27, 425-436.

15. George A.; Romaguera, S. Characterizing completable fuzzy metric spaces. Fuzzy Set. Syst. 2004, 144, 411-420. 
16. Hong, S.M.; Park, S.-J.; Jeong, J. Deinterlacing algorithm using fixed directional interpolation filter and adaptive distance weighting scheme. SPIE Opt. Eng. 2011, 50, doi:10.1117/1.3584843.

(C) 2013 by the authors; licensee MDPI, Basel, Switzerland. This article is an open access article distributed under the terms and conditions of the Creative Commons Attribution license (http://creativecommons.org/licenses/by/3.0/). 